\title{
THE MAJOR HISTOCOMPATIBILITY COMPLEX: A REVIEW
}

\author{
PREETI SHARMA ${ }^{1 *}$, PRADEEP KUMAR ${ }^{1}$, RACHNA SHARMA $^{2}$
}

${ }^{1}$ Department of Biochemistry, Santosh Medical College and Hospital, Ghaziabad, Uttar Pradesh, India. ${ }^{2}$ Department of Biochemistry, TSM Medical College and Hospital, Lucknow, Uttar Pradesh, India. Email: prcdri2003@yahoo.co.in

Received: 06 October 2016, Revised and Accepted: 28 October 2016

ABSTRACT

One of the important components of the immune system, the major histocompatibility complex (MHC) molecules allow T-lymphocytes to detect cells, such as macrophages, B-lymphocytes, and dendritic cells that ingest infectious microorganisms or the self-cells infected with microorganism. On being engulfed a microorganism, macrophage partially digests it and displays peptide fragments of the microbe on its surface, bound to MHC molecules and the T-lymphocyte recognizes the foreign fragment attached to the MHC molecule and binds to it, lead to stimulation of an immune response. The MHC molecule presents peptides from its own cell (self-peptides) in healthy self-cells to which T-cells do not normally react.

Keywords: MHC, B Cells, T Cells, Antigen Processing.

(c) 2017 The Authors. Published by Innovare Academic Sciences Pvt Ltd. This is an open access article under the CC BY license (http://creativecommons. org/licenses/by/4. 0/) DOI: http://dx.doi.org/10.22159/ajpcr.2017.v10i2.15555

\section{INTRODUCTION}

MHCs are a class of molecules that influence the capability of an organism to accept or reject transplanted tissues. In contrast to the B-cell receptors and antibodies which recognize the antigen single handedly, T-cell receptor (TCR) only recognizes the antigen processed and presented by MHC molecules [1,2]. The functions of MHC molecules include its binding to a peptide fragments derived from some pathogenic microorganism. Display of these antigens happens on the cell surface for its recognition by some suitable T-cells [3]. These cause deleterious effects on the pathogen, virus-infected cells are killed, and macrophages are also induced for killing bacteria to other pathogens living in their intracellular vesicles [3]. Parallel to these happenings, B-cells are activated to generate antibodies that remove or neutralize extracellular pathogens [4]. In course of all these events, there are chances in favor of any pathogen that it has mutated in such a way so as to escape from presentation by an MHC molecule [5]. MHC molecules decide upon the response of an individual to antigenic infection. Thus, its role is implicated in the susceptibility to the disease as well as in autoimmune disease [6]. "Human leukocyte antigen" is the expanded version of "HLA," which is similar to MHC in structure and function, is a group of antigens or proteins that are found at the surface of cells and in the genetic makeup or DNA [7]. A T-cell recognizes peptide antigen which is attached by a specific kind of allelic variant of an MHC molecule. It will not recognize the same peptide bound to other MHC molecules and this quality of T-cells is called MHC restriction [8]. They are the biological tools meant to identify and prevent a foreign protein or cell in entering or its spreading in an organism's body [2]. Being one of the important components of immune system, these proteins recognize between self and nonself $[2,3]$. The MHC molecules are often present in vertebrates while HLA is found only in humans and it can be better explained as the human body's version of MHC [9]. Local cells carrying viruses and other harmful organisms are often identified with the help of these MHC or HLA molecules and attacked [2]. Many times these antigenic molecules are involved when an organ transplant is planned for an organism or a human being. Some tests are carried out to determine the compatibility between an organ and a recipient's body and near perfect or perfect matches are desirable in these situations to lessen the risk and also the chances of the recipient's body in organ rejection [10]. Therefore, scope of both MHC and HLA in organ transplants lies in making our body and its defense stronger. Besides its important role in immunity, HLA is also used in determination of the parentage of a child by comparing antigens from the child, father, and mother [11] in paternity testing. Earlier studies have shown that these MHCs are derived from a cluster of genes and play an important role in both cell mediated and humoral immunity [2-4]. These clustered genes lie on long continuous stretch of DNA on the chromosome 6 among the human beings and it is on chromosome 17 in mice [12]. In humans, they are also known by the name HLA complex [12]. MHC gene encodes for 3 major and important classes of molecules which are Class 1, 2, and 3. Class I MHC genes encode a particular glycoproteins on every nucleated cell of the body and major played by this class of molecule is the presentation of peptide antigens to $\mathrm{T}_{\mathrm{c}}$ (cytotoxic T-cells) while Class II MHC genes encode for the glycoproteins, which are expressed only on the surface of antigen presenting cells (APCs) such as macrophages, dandritic cells, and B-cells [13]. Here, antigenic peptides after being processed are presented to Th-cells. Class III MHC gene encodes various proteins that play important immune role, and also the components of complement system including proteins involved in inflammation are being mediated by these [14]. The Class I and II molecules have important roles in antigen processing and presentation [13] while Class III region which is flanked by Class I and II regions in the gene is responsible for the expression of the molecules concerned with immune function [14]. There are number of complement factors including C2, C4, factor $\mathrm{B}$, and several inflammatory cytokines including tumor necrosis factor which is expressed by the mediation of Class III MHCs [14].

\section{MHC CLASS I MOLECULES}

Structurally, MHC Class I molecules are heterodimers. It consist of two polypeptide chains, $\alpha$ and $\beta 2$-microglobulin $(\mathrm{b} 2 \mathrm{~m})$ as heavy and light chain, respectively [15]. The two chains are linked noncovalently through interaction of $\mathrm{b} 2 \mathrm{~m}$ and $\alpha 3$ domain. The $\alpha$-chain is polymorphic in character and encoded by a HLA gene while the b2m subunit is not polymorphic and encoded by the b2m gene [15]. The $\alpha 3$ domain spans over the plasma membrane and interacts with the CD8 coreceptor of T-cells and this interaction holds the MHC I molecule in place [15]. The other kind of receptor called TCR lies on the surface of the cytotoxic T-cell binds its $\alpha 1-\alpha 2$ heterodimer ligand and checks the coupled peptide for antigenicity [15]. The $\alpha 1$ and $\alpha 2$ domains fold in a manner to make up a groove for peptides (self or nonself) to bind. MHC Class I molecules bind peptides that are 8-10 amino acid in length [16]. The peptide (self or nonself) translocation from the cytosol into the lumen 
of the endoplasmic reticulum (ER) is accomplished by the transporter associated with antigen processing (TAP) which is a member of the ABC transporter family and is a heterodimeric multimembrane-spanning polypeptide consisting of TAP1 and TAP2 $[15,16]$. The two subunit forms of TAP are peptide binding site and two ATP binding sites that face the cytosol (Fig. 3). The antigenic or nonantigenic peptides are now bonded with TAP on the cytoplasmic side and translocate them by consuming ATPs into the lumen of the ER and then, the MHC Class I molecule is in turn loaded with peptides in the lumen of the ER. This complex phenomenon of peptide-loading involves several other molecules that form a large multimeric complex consisting of TAP, tapasin, calreticulin, calnexin, and Erp57 [16]. Calnexin acts to stabilize the Class I MHC $\alpha$ chains before $\beta 2 \mathrm{~m}$ binding $[16,17]$. Following complete assembly of the MHC molecule, calnexin dissociates. The MHC molecule lacking a bound peptide is inherently unstable and requires the binding of the chaperones calreticulin and Erp57 [16]. The protein tapasin binds to the MHC molecule to link it to the TAP proteins, to facilitate enhanced peptide loading and colocalization.

On being loaded with the peptide, the MHC Class I molecule, the complex dissociates and it leaves the ER through the secretory pathway to reach the cell surface. The transport of the MHC Class I molecules through the secretory pathway involves several posttranslational modifications of the MHC molecule, involving change to the N-glycan regions of the protein, followed by extensive changes to the $\mathrm{N}$-glycans in the Golgi apparatus. The N-glycans' form is the completely mature form before they reach the cell surface. Fig. 1 shows the processing and presentation of the antigen by MHC Class I molecule [17].

\section{MHC CLASS II MOLECULES}

MHC Class II consists of two polypeptide chains, $\alpha$ and $\beta$, each having two domains $\alpha 1$ and $\alpha 2$ and $\beta 1$ and $\beta 2$. Each chain is having a transmembrane domain, $\alpha 2$ and $\beta 2$, respectively, for attaching the MHC Class II molecule to the cell membrane [19]. The peptide-binding groove is consisted of the heterodimer of $\alpha 1$ and $\beta 1$. MHC Class II molecules are normally occur only on professional APCs such as macrophages, B-cells, and especially dendritic cells (DCs) [20]. Although they can be conditionally expressed by all cell types. An APC takes up an antigen, performs antigen processing, and returns a molecular fraction of it termed as the epitope to the APC's surface, coupled within an MHC Class II molecule. The process is known as antigen presentation [20]
(Fig. 4). In this unique presentation, the epitopes are recognized by Th-cells [19]. The CD4 receptors as well as TCRs are present on the surfaces of helper T-cells. When a CD4 molecule of a naive helper T-cell links to an APC's MHC Class II molecule, its TCR can meet the epitope coupled within the MHC Class II, the phenomenon similar to the coupling of $T_{c}$ with cytotoxic cells [19]. This event primes the naive helper T-cell. The varied combination of cytokines secreted by APCs in the microenvironment causes the naive helper T-cell to polarize into either a memory Th-cell or an effector Th-cell [19].

MHC Class II thus mediates immunization to APCs, differentiate the T-cell into memory, and effector cells so that in future if the same antigen invades the organism, the memory cells get activated and trigger the immune response [20]. The polarization during primary exposure to an antigen is key in determining a number of chronic diseases, including inflammatory bowel diseases and asthma, by inducing the immune response that is memory Th-cells coordinate when their memory recall is triggered upon secondary exposure to similar antigens [21].

In the phase of processing and presentation of the antigen, the phagocytes such as macrophages and immature dendritic cells take up antigenic entities by phagocytosis into phagosomes [22]. B-cells exhibit the more general endocytosis into endosome [23]. These phagosomes or endosomes fuse with lysosomes [22]. Its acidic enzymes cleave the uptaken protein into many different peptides. Out of these, a particular peptide exhibits immunodominance and loads onto MHC Class II molecules. APCs express both kind of MHC I \& II molecules (Fig. 2). However, in the cell, there is highly advanced system exists to prevent Class II molecules from binding to same set of antigenic pepides as the Class I molecules [24]. In course of synthesis of MHC II in the ER, three pairs of Class II $\alpha \beta$ chains associate with a preassembled protein (trimeric) called invariant chain which interacts with peptide binding cleft of the MHC Class II molecules block the attachment of any endogenous peptide to the cleft [20]. The invariant chain complex with Class II molecule is now transported from the RER through golgi complex to endosome-lysosome assembly. During the journey from RER to the endosome-lysosome assembly, the invariant chain gets degraded under the influence of proteolytic enzymes and a small fragment CLIP (Class II-associated invariant chain peptide) remains attached to the Class II cleft [25]. For exchange of CLIP with antigenic

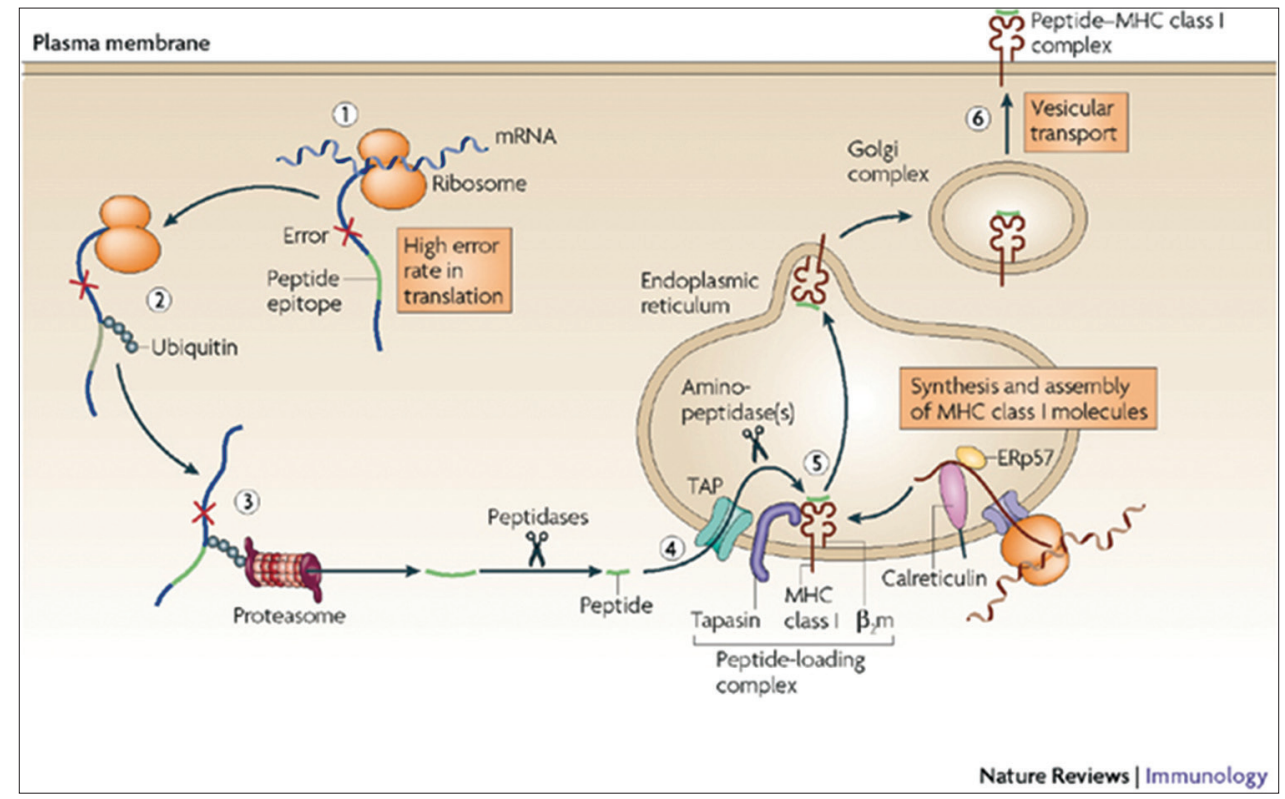

Fig. 1: Antigen processing and presentation by major histocompatibility complex Class I molecule [18] 
peptide, there is requirement of nonclassical Class II molecule which is called HLA-DM. It is a heterodimer but not polymorphic and is not expressed at the cell membrane. HLA-DM is found predominantly in the endosomal compartment. The reaction between HLA-DM and Class II CLIP complex facilitates the exchange of CLIP for another peptide. Now, the MHC Class II molecule uploaded with the antigenic peptide is trafficked to and externalized on the cell surface [26].

\section{MHC MULTIMERS}

The tailormade various oligomeric forms of MHC molecules have been designed to identify and isolate T-cells with high affinity to specific antigens amid a large group of unrelated T-cells are known as MHC multimers. TCRs have a low affinity for their MHC counterparts. Initially,

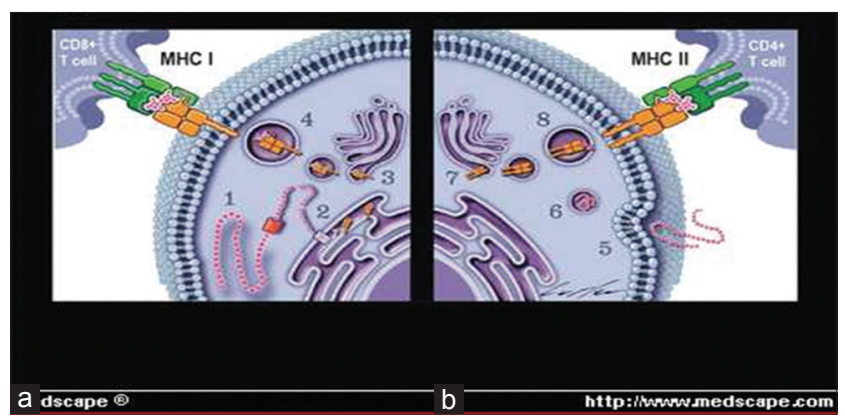

Fig. 2: ( $a$ and $b$ ) Comparative account of antigen processing and presentation by major histocompatibility complex (MHC) Class I and MHC Class II molecule [18]

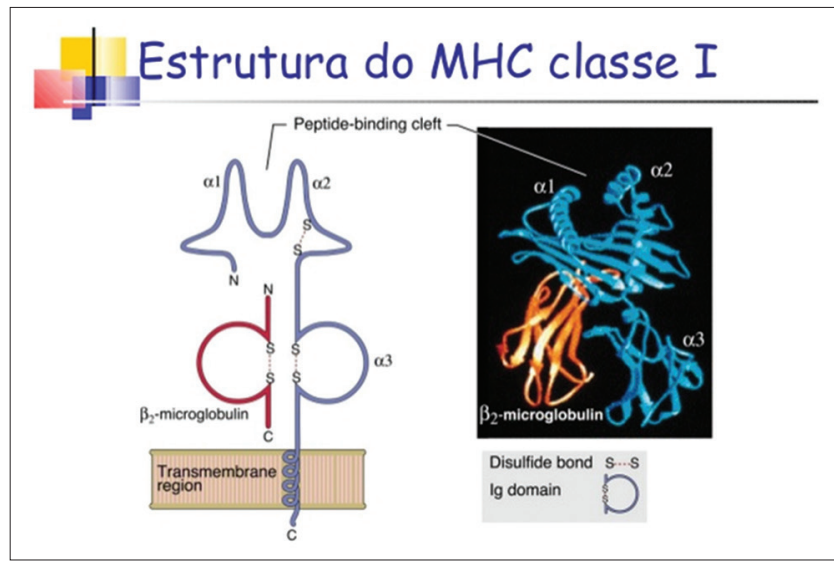

Fig. 3: Structure of major histocompatibility complex Class I molecule [18] it was problematic to label T-cells effectively using single MHC-T-cell interactions. In 1996, John Altman used a complex of multiple MHC molecules to form a more stable bond between corresponding T-cells and this gave birth to the MHC multimers [27].

These multimeric molecules may range in size from dimers to octamers. Currently, few companies are using even higher quantities of MHC per multimer to increase their specificity. These molecules are used to display Class $1 \mathrm{MHC}$, CLASS $2 \mathrm{MHC}$, or nonclassical molecules such as CD1d from species such as monkeys, mice, and humans [27]. The most commonly used MHC multimers are tetramers which are typically produced by biotinylating soluble MHC monomers. Soluble MHC monomers are typically produced recombinantly in eukaryotic or bacterial cells and they then bind to a backbone, such as streptavidin or avidin, creating a tetravalent structure [28]. These backbones are conjugated with fluorochromes to isolate bound T-cells through flow cytometry technique. MHC multimers are of use in antigen-specific T-cell detection and isolation and their ability gives rise to several clinical applications. MHC multimers have broadened the strategy for ex vivo selection and proliferation of T-cells specific to viral or tumor-related antigens. These T-cells can then be reintroduced to augment the immune system. Beside its vast scope in T-cell selection and proliferation, MHC multimers can also be used to eliminate graft-originating T-cells on transplant organs, ex vivo and in vivo; these MHC multimers may also be used to eliminate harmful or unwanted T-cells, such as those that target self-cells and lead to autoimmune disease. We can do desirable modifications in cancer immunotherapy and vaccine development by involving MHC multimers [29].

\section{CONCLUSION}

The two major classes of MHC protein molecules are Class I and Class II. Class I MHC molecules span the membrane of almost every nucleated cell in an organism. On the other hand, class II molecules are concerned with cells of the immune system known as macrophages, dendritic cells, and lymphocytes. In human beings, these molecules are encoded by several genes which are clustered in the same region on chromosome 6 and each gene has an unusually large number of alleles which are actually alternate forms of a gene producing alternate forms of the proteins. Hence, rarely any two individual have the same type of set of MHC molecules, which are jointly known as tissue type. The MHC class III genes are so diverse and they encode for other proteins such as complement proteins, cytokines (chemical messengers), and enzymes. Besides multimer, MHC molecules have diverse scope in the field of clinical medicine and research. They can also be used to remove or arrest graft-originating T-cells due to organ transplant, ex vivo. In vivo, these MHC multimers may also be used to eliminate harmful or unwanted T-cells that lead to autoimmune disease.

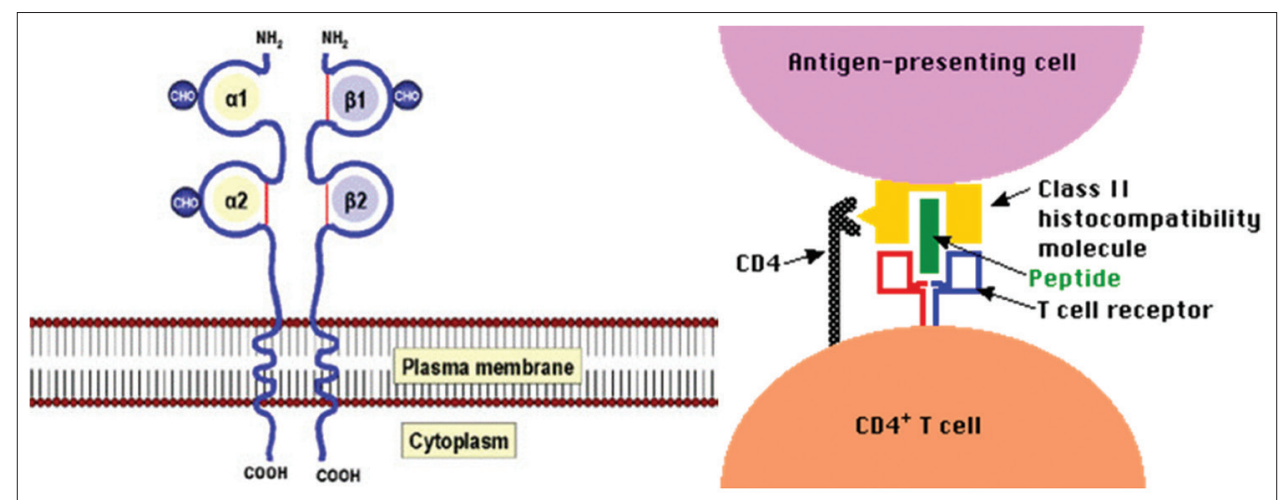

Fig. 4: Structure of major histocompatibility complex Class II molecule and interaction between antigen presenting cell and T-cell with its T-cell receptor and coreceptor $\mathrm{CD}_{4}[18]$ 


\section{REFERENCES}

1. Janeway CA Jr, Travers P, Walport M, Shlomchik MJ. the major histocompatibility complex and its functions. Immunobiology: The Immune System in Health and Disease. $5^{\text {th }}$ ed. New York: Garland Science; 2001.

2. Complete sequence and gene map of a human major histocompatibility complex. The MHC sequencing consortium. Nature 1999;401(6756):921-3.

3. Abbas AK, Lichtman AH. Antigen capture and presentation to lymphocytes. Basic Immunology: Functions and Disorders of the Immune System. $3^{\text {rd }}$ ed. Ch. 3. Philadelphia, PA: Saunders; 2009.

4. Kindt TJ, Goldsby RA, Osborne BA, Kuby J. Kuby Immunology. Macmillan, 2007. ISBN 978-1-4292-0211-4. Available from: https:// en.wikipedia.org/wiki/Major histocompatibility_complex. [Last retrieved on 2010 Nov 28].

5. Batista FD, Harwood NE. The who, how and where of antigen presentation to B cells. Nat Rev Immunol 2009;9(1):15-27.

6. Fogdell-Hahn A, Ligers A, Gronning M, Hillert J, Olerup O. Multiple sclerosis: A modifying influence of HLA class I genes in an HLA class II associated autoimmune disease. Tissue Antigens 2000;55(2):140-8.

7. Mungall AJ, Palmer SA, Sims SK, Edwards CA, Ashurst JL, Wilming L, et al. The DNA sequence and analysis of human chromosome 6. Nature 2003;425(6960):805-11.

8. Murphy K. Antigen recognition by $\mathrm{T}$ cells. In: Janeway's Immunobiology. $8^{\text {th }}$ ed. New York: Garland Science; 2012. p. 138-53.

9. Available from: https://www.en.wikipedia.org/wiki/Human_leukocyte_ antigen.

10. Chandraker A, Lacomini JJ. Transplantation immunobiology. In: Brenner BM, Leivne SA, editors. Brenner \& Rector's the Kidney. $8^{\text {th }}$ ed. Philadelphia, PA, USA: Elsevier, Saunders; 2007. p. 2104-11.

11. Elston RC. Probability and paternity testing. Am J Hum Genet 1986;39(1):112-22.

12. Strachan T, Read A. Human chromosome groups. Human Molecular Genetics. $2^{\text {nd }}$ ed. New York and London: Garland Science; 1999

13. Kambayashi T, Laufer TM. Atypical MHC class II-expressing antigenpresenting cells: Can anything replace a dendritic cell? Nat Rev Immunol 2014;14(11):719-30.

14. Gruen JR, Weissman SM. Evolving views of the major histocompatibility complex. Blood 1997;90(11):4252-65.

15. Albring J, Koopmann JO, Hämmerling GJ, Momburg F.
Retrotranslocation of MHC class I heavy chain from the endoplasmic reticulum to the cytosol is dependent on ATP supply to the ER lumen. Mol Immunol 2004;40(10):733-41.

16. Hewitt EW. The MHC class I antigen presentation pathway: Strategies for viral immune evasion. Immunology 2003;110(2):163-9.

17. Koopmann JO, Albring J, Hüter E, Bulbuc N, Spee P, Neefjes J, et al. Export of antigenic peptides from the endoplasmic reticulum intersects with retrograde protein translocation through the Sec61p channel. Immunity 2000;13(1):117-27.

18. Available from:http://www.google.co.in/search?q=structure + of + mhc + class.

19. Babbitt BP, Allen PM, Matsueda G, Haber E, Unanue ER. Binding of immunogenic peptides to Ia histocompatibility molecules. Nature 1985;317(6035):359-61.

20. Castellino F, Zhong G, Germain RN. Antigen presentation by MHC class II molecules: Invariant chain function, protein trafficking, and the molecular basis of diverse determinant capture. Hum Immunol 1997;54(2):159-69.

21. Whitmire JK, Eam B, Whitton JL. Tentative T cells: Memory cells are quick to respond, but slow to divide. PLoS Pathog 2008;4(4):e1000041.

22. Savina A, Amigorena S. Phagocytosis and antigen presentation in dendritic cells. Immunol Rev 2007;219:143-56.

23. Iwasaki A, Pillai PS. Innate immunity to influenza virus infection. Nat Rev Immunol 2014;14(5):315-28.

24. Available from: https://www.ncbi.nlm.nih.gov/books/NBK26926.

25. Available from: https://www.en.wikipedia.org/wiki/Antigen processing.

26. Chaturvedi P, Yu Q, Southwood S, Sette A, Singh B. Peptide analogs with different affinites for MHC alter the cytokine profile of T helper cells. Int Immunol 1996;8(5):745-55.

27. Hadrup SR, Bakker AH, Shu CJ, Andersen RS, van Veluw J, Hombrink $\mathrm{P}$, et al. Parallel detection of antigen-specific T-cell responses by multidimensional encoding of MHC multimers. Nat Methods 2009;6(7):520-6.

28. Bakker AH, Schumacher TN. MHC multimer technology: Current status and future prospects. Curr Opin Immunol 2005;17(4):428-33.

29. Lebowitz MS, O'Herrin SM, Hamad AR, Fahmy T, Marguet D, Barnes NC, et al. Soluble, high-affinity dimers of T-cell receptors and class II major histocompatibility complexes: Biochemical probes for analysis and modulation of immune responses. Cell Immunol 1999;192(2):175-84 